\title{
AGO Recommendations for the Diagnosis and Treatment of Patients with Early Breast Cancer: Update 2014
}

\author{
Cornelia Liedtke $^{a} \quad$ Marc Thill $^{b} \quad$ Volker Hanf $^{c} \quad$ Florian Schütz $^{d}$ \\ on behalf of the AGO Breast Committee \\ ${ }^{a}$ Klinik für Frauenheilkunde und Geburtshilfe, Universitätsklinikum Schleswig Holstein, Campus Lübeck,

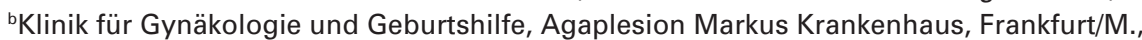 \\ cFrauenklinik, Klinikum Fürth, \\ ${ }^{\mathrm{d}}$ Frauenklinik, Universitätsklinikum Heidelberg, Germany
}

\section{Keywords}

Breast cancer - Treatment recommendations - Surgery . Adjuvant treatment

\section{Introduction}

For the last 13 years, the Breast Committee of the Arbeitsgemeinschaft Gynäkologische Onkologie (German Gynecological Oncology Group, AGO) has been preparing and updating evidence-based recommendations for the diagnosis and treatment of patients with early and metastatic breast cancer. The AGO Breast Committee consists of gynecological oncologists specialized in breast cancer and interdisciplinary members specialized in pathology, radiological diagnostics, medical oncology, and radiation oncology. This update has

Members of the 'AGO Breast Committee' in alphabetical order: UteSusan Albert, Marburg; Ingo Bauerfeind, Landshut; Joachim Bischoff, Magdeburg; Jens Uwe Blohmer, Berlin; Klaus Brunnert, Osnabrück; Peter Dall, Lüneburg; Ingo J. Diel, Mannheim; Tanja Fehm, Düsseldorf; Nikos Fersis, Bayreuth; Michael Friedrich, Krefeld; Kay Friedrichs, Hamburg; Bernd Gerber, Rostock; Volker Hanf, Fürth; Nadia Harbeck, München; Jens Huober, Ulm; Christian Jackisch, Offenbach; Wolfgang Janni, Ulm (Co-Chair); Hans H. Kreipe, Hannover (DGP); Thorsten Kühn, Esslingen; Sherko Kümmel, Essen; Cornelia Liedtke, Lübeck; Sibylle Loibl, Neu-Isenburg; Hans-Joachim Lück, Hannover; Michael Lux, Erlangen; Nicolai Maass, Aachen; Gunter von Minckwitz, NeuIsenburg; Volker Möbus, Frankfurt; Christoph Mundhenke, Kiel; Volkmar Müller, Hamburg; Ulrike Nitz, Mönchengladbach; Mahdi Rezai, Düsseldorf; Achim Rody, Lübeck; Anton Scharl, Amberg (Chair); Rita Schmutzler, Köln; Marcus Schmidt, Mainz; Andreas Schneeweiss, Heidelberg (AIO); Ingrid Schreer, Hamburg (DGS); Florian Schütz, Heidelberg; Peter Sinn, Heidelberg (Pathologie); Erich F. Solomayer, Homburg; Rainer Souchon, Tübingen (ARO); Elmar Stickeler, Freiburg; Marc Thill, Frankfurt; Christoph Thomssen, Halle (Saale); Michael Untch, Berlin. been performed according to a documented rule-fixed algorithm, by thoroughly reviewing and scoring chapter by chapter the recent publications for their scientific validity (Oxford level of evidence (LoE), www.cebm.net [1]) and clinical relevance (AGO grades of recommendation (GR); table 1). We present the 2014 update; the full version of the updated slide set is available online as a PDF file in both English and German [2].

\section{Breast Cancer Risk Factors}

Individual risk factors can be classified into non-modifiable, modifiable, and socially defined factors. Currently, there is good evidence that changes in some modifiable risk factors could substantially decrease the breast cancer risk. This means that every woman could decrease her personal risk of breast cancer by adopting a healthy lifestyle.

With the exception of a total fat intake that might increase the body mass index (BMI) and thus the breast cancer risk, there is no convincing data that the intake of fruits and vegetables, micronutrients, trace elements, or vitamins may reduce the breast cancer risk [3, 4]. However, prevention of diabetes mellitus type II could reduce breast cancer incidence and mortality [5].

Alcohol use may be more strongly associated with the risk of hormone-sensitive breast cancers than of hormone-insensitive subtypes, suggesting distinct etiologic pathways for these two breast cancer subtypes [6].

A systematic review and meta-analysis on the association of oral contraceptives (OC) and risk of ovarian cancer and breast cancer among high-risk women (BRCA mutation carriers) suggest that associations between ever-use of OC and ovarian and breast cancer among women who are BRCA1 or BRCA2 mutation carriers are similar to those reported for the general population [7].

\section{KARGER}

Fax +497614520714

Information@Karger.com

www.karger.com (c) 2014 S. Karger GmbH, Freiburg

1661-3791/14/0093-0189\$39.50/0

Accessible online at:

www.karger.com/brc
PD Dr. med. Cornelia Liedtke

Klinik für Frauenheilkunde und Geburtshilfe

Universitätsklinikum Schleswig-Holstein/Campus Lübeck

Ratzeburger Allee 160, 23538 Lübeck, Germany

cornelia.liedtke@uksh.de 
Table 1. AGO grades of recommendation

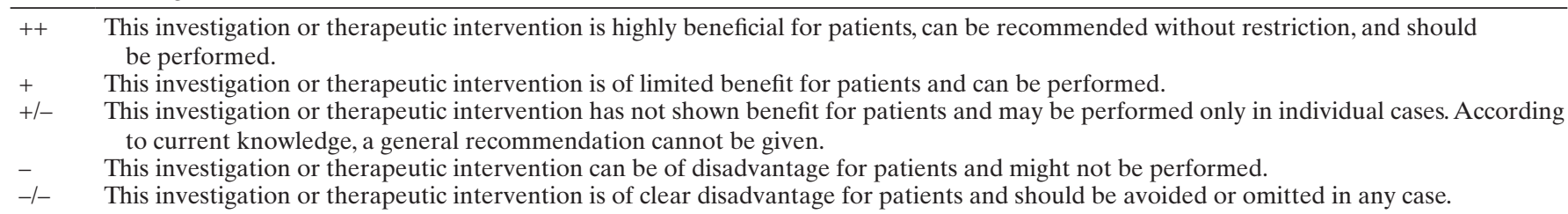

\section{Hereditary Breast Cancer and Prevention}

The indication of testing patients for BRCA1/2 mutations is based on family and personal histories of breast and/or ovarian cancer. However, before performing genetic testing, counselling and informed consent is mandatory and should cover the consequences after the detection of a mutation.

A checklist regarding the personal history is available in German (www.aekwl.de/fileadmin/qualitaetssicherung/ Zertifizierungsstelle/FB-erbliche-Belastung-V2013-08-07.pdf).

Of all genetic testing results, 5-30\% reveal variants of unknown significance (VUS), of which it is not clear if they are causative for disease development [8]. As more than $60 \%$ of the VUS are confidential or extremely rare and population specific, only large databases such as the database of the German Consortium of Hereditary Breast and Ovarian Cancer (GC-HBOC) allow the classification of most of these VUS. BRCA1 and BRCA 2 explain only about $50 \%$ of the familial aggregation. Recent data suggest that no further high-risk genes such as BRCA1/2 (odds ratio $(\mathrm{OR})>5.0$ ) exist and that the remaining heritability is due to moderate-risk genes (e.g. RAD51C, ATM, BRIP1, CDH1, CHEK2, NBN, PALB2, PTEN; OR 1.5-5.0) and low-risk variants (FGFR2, TOX3, 2q35, 11q15, SLC4A7, 5p12, MAP3K1; OR < 1.5) that are transmitted via an oligogenetic trait [9-11]. Low-risk variants also show associations with specific breast cancer subtypes [12]. RAD51C is a new moderate-to-high-risk gene. Moreover, there are many non-BRCA-associated hereditary cancer syndromes with an increased risk for breast cancer (LiFraumeni, Cowden, hereditary diffuse gastric cancer syndrome, Peutz-Jeghers syndrome, Lynch syndrome). The use of commercially available but not validated breast cancer gene panels for risk prediction is not recommended outside of controlled clinical trials [13].

For many of those genetically defined subtypes, issues such as histopathological features, sensitivity to different screening modalities, course of disease, or specific treatment response still remain unclear. Healthy women who are identified as being at moderate to high risk for disease development should be offered participation in a surveillance/screening program. Women with BRCA1/2 mutations should also be offered nondirective counselling for the uptake of primary preventive measures (e.g. risk-reducing bilateral salpingo-oophorectomy at around 40 years of age, risk-reducing bilateral mastectomy, or medical prevention with tamoxifen, raloxifen, or an aromatase inhibitor) in addition to participation in a surveillance/ screening program. Until now there are no specific treatment options in diseased mutation carriers. Breast-conserving surgery (BCS) is safe and the use of platinum-based chemotherapy regimens as well as poly(ADP-ribose) polymerase (PARP) inhibitors is currently validated in prospective studies.

\section{Breast Cancer Diagnostics}

The aim of early detection and screening of breast cancer is to reduce the breast cancer specific mortality and the treatment-dependent morbidity. The detection of invasive breast cancer at an early stage (stage I-IIA) offers the chance to survive this disease with less treatment impairment and better quality of life.

Professionals and women need to be informed about the benefits and harms of cancer screening tests before making medical decisions. This includes clear and understandable information in absolute terms about false-positive rates, falsenegative rates, overdiagnosis, and overtreatment.

Supplemental breast ultrasound (US) in the population of women with mammographically dense breast tissue (American College of Radiology (ACR) 3 and 4) permits the detection of small, otherwise occult, breast cancers. Potential adverse impacts on women in the intermediate-risk group are associated with an increased biopsy rate. The arguments against US used as a screening modality alone are lack of reproducibility, high false-positive rate, low positive predictive value (PPV) for biopsy, inability to detect most ductal carcinoma in situ (DCIS) cases, operator dependency, and lack of quality assurance.

There are no data to recommend breast US alone or automated breast volume scanning (ABVS) as breast cancer screening methods. The recent Cochrane Database Systematic Review from 2013 [14] 'did not detect any controlled studies on the use of adjunct ultrasonography for screening in women at average risk for breast cancer. One ongoing randomized controlled trial was identified ...' That is why 'presently, there is no methodologically sound evidence available, justifying the routine use of ultrasonography as an adjunct screening tool in women at average risk for breast cancer.'

For BRCA mutation carriers, an additional (to breast magnetic resonance imaging (MRI) and mammography) semi-annual US screening seems to be a sensitive and effective method $[15,16]$. Elastography is a US-based method and has some advantages in diminishing the rate of Breast Imag- 
ing Report and Data System (BI-RADS) III lesions and in measuring the true size of breast cancer lesions [17].

According to a recent meta-analysis, the re-excision rate is not reduced but the initial and total rate of mastectomy is increased if a preoperative breast MRI is performed compared with no preoperative breast MRI $[18,19]$. In case of lobular invasive breast cancer, there is a significant reduction of the re-excision rate and no significant impact on the rate of mastectomies due to the use of preoperative breast MRI $[18,19]$. According to the second meta-analysis of this study group [19], the preoperative breast MRI does not reduce the rate of local recurrences and does not improve the local recurrencefree survival and the distant metastases-free survival [18]. This is why preoperative breast MRI is not recommended as a routine method for all patients. For some patients, e.g. with high breast density (ACR 3-4) and lobular invasive cancer, it can be considered ( $\mathrm{LoE} 1 \mathrm{~b}, \mathrm{~B}, \mathrm{AGO}++$ ).

With the use of intraoperative US, the rate of re-excisions, the volume of resection, and the rate of involved margins are significantly reduced according to a randomized study [20] and a recent meta-analysis [21]. That is why intraoperative US can be performed as a method to reduce the re-excision rate and rate of $\mathrm{R} 1$ resections ( $\mathrm{LoE} 1 \mathrm{a}, \mathrm{A}, \mathrm{AGO}+/-$ ).

If there is a clinical and/or sonographical suspicious axillary lymph node, US-guided fine needle aspiration or core cut biopsy may be performed to avoid two-stage axillary surgery $(\mathrm{LoE} 2 \mathrm{~b}, \mathrm{~B}, \mathrm{AGO}+)^{2}$ [2]. The standard procedure in patients with unsuspicious axillary lymph nodes is sentinel node biopsy.

Mammography, breast US, and breast MRI are the preferred methods for evaluating the response to neoadjuvant chemotherapy (NACT). Until now, breast MRI seems to be the best method to predict pathologic complete response (pCR) after NACT. According to an indirect comparison between MRI and breast US, US seems to have the same sensitivity and specificity to predict pCR after NACT [21, 23-26]. Definition standards of response are required. The PPV for predicting pCR ranges around $47-73 \%$, and the negative predictive value (NPV) around 71-100\%. First results with diffusion-weighted (DW)-MRI show that the pretreatment apparent diffusion coefficient (ADC) is dependent on the tumor subtype [27].

Up to now, none of the imaging modalities are predictive of pCR.

\section{Pathology}

Because of growing evidence that a considerable proportion of human epidermal growth factor receptor 2 (HER2)positive cases may be overlooked if the testing is primarily based on the ratio of the HER2 gene copy number in relation to centromere 17, a new consensus on HER2 testing was published by the American Society of Clinical Oncology (ASCO)/
College of American Pathologists (CAP) [28]. Accordingly, the AGO guidelines have been revised with some modifications in comparison to the published consensus. The essential changes are:

- Equivocal cases by in situ hybridization (ISH) are no longer defined by ratio but exclusively by an average copy number of $\geq 4$ to $<6$ HER 2 signals/nucleus.

- Even with a ratio $<2$, cases can be HER2 positive if $\geq 6$ HER2 signals are present.

- Cases with an average copy number of $\geq 4$ to $<6$ HER2 signals/nucleus (equivocal), or $<4$ signals but with a ratio $\geq 2$, are considered as HER2 positive.

- Less than $10 \%$ strongly membrane-stained cells by immunohistochemistry (IHC) are considered as HER2 2+ (equivocal cases requiring additional testing by ISH).

- In rare subtypes (e.g. micropapillary carcinoma), the HER2 gene may be amplified but IHC reveals incomplete (U-shaped) staining. In these rare cases, incomplete staining as an exception from the rule has to be considered as $2+$, requiring additional testing.

With regard to the latter aspect, the AGO Breast Committee did not follow the published consensus, which does not require completeness of membrane staining as a $2+$ criterion anymore. In fact, the AGO considers it more appropriate to exemplify the rare exceptions in which the HER2 gene might be amplified despite incomplete membrane staining, namely micropapillary carcinoma. Thus, reflex ISH should be done

- when the traditional Food and Drug Administration (FDA)-accepted 2+ criteria are met,

- in those rare cases with incomplete moderate-to-strong membrane staining of special histological types, and

- in tumors with strong and complete staining in $<10 \%$ of cells. Otherwise, many of the former IHC $1+$ cases with weak/moderate incomplete staining would be analyzed by ISH, unnecessarily increasing the costs for diagnostic HER2 testing [29].

$\mathrm{Ki}-67$ is helpful in determining the grade of tumors. Grade 1 (G1) tumors usually show a Ki-67 index below $15 \%$ and G3 tumors exhibit a labeling index $\geq 25 \%$. In core biopsies, Ki-67 is better suited to predict the final histological grade than mitotic counts. There are a number of studies stating that a threshold of $20 \%$ is able to discriminate between the luminal A and B types, but further confirmation should be awaited. Reproducibility and interest and interlaboratory variation of testing are important issues if the Ki-67 growth index is determined and utilized for clinical decision-making

\section{Prognostic and Predictive Factors}

The AGO Breast Committee acknowledges that 2 kinds of criteria for the validation and grading of evidence regarding clinical significance are valid for prognostic and predictive factors: (1) the Oxford LoE (LoEOx2001) criteria and the 
GR, which are used throughout the guidelines, and (2) the GR as well as the modified LoE criteria for use in archived specimens (LoE2009) and in the category of tumor marker study (CTS) [30]. It needs to be emphasized that the 2 evidence levels cannot be directly compared. In particular, the prospectively planned retrospective validation of a biomarker may be severely biased by the number of samples available for biomarker analysis from the original trial. Since the optimal percentage of samples needed has not yet been determined, the AGO commission reports the original percentages in their slide set so that the readers can draw their own conclusions about the quality of the available data.

In node-negative breast cancer, the urokinase-type plasminogen activator (uPA)/plasminogen activator inhibitor-1 (PAI-1) enzyme-linked immunosorbent assay (ELISA) (Femtelle $^{\circledR}$ ) is a recommended ( $\mathrm{LoE} 1 \mathrm{a}, \mathrm{A}, \mathrm{AGO}+$ ) prognostic and predictive factor for the decision regarding adjuvant chemotherapy [31]. In doubtful cases of node-negative endocrine-responsive HER2-negative tumors, a multigene assay such as Oncotype DX ${ }^{\circledR}$ or Endopredict ${ }^{\circledR}$ may be helpful in the decision-making process to opt for endocrine therapy alone versus the chemoendocrine treatment option (LoE2009 I, B, $\mathrm{AGO}+$ ). In node-positive patients, the use of these assays needs to be carefully discussed with the patients (LoE2009 II, $\mathrm{B}, \mathrm{AGO}+/-)$ since the prospective clinical trials in this setting (Rxponder, WSG-ADAPT, WSG-Plan B) have not yet been completed and reported.

The determination of molecular subtypes in paraffin-embedded tissue has now become available using the validated PAM50 (Prosigna ${ }^{\circledR}$ ) assay (LoE2009 II, B, AGO +/-) [32]. Prosigna [33] and Endopredict ${ }^{\circledR}$ [34] also provide information about late recurrences, which may be used to guide endocrine therapy for more than 5 years.

Circulating tumor cells (CTCs; Cell Search ${ }^{\circledR}$ ) can be used only in the metastatic setting as a prognostic tool (LoE2009 I, $\mathrm{B}, \mathrm{AGO}+$ ) since their prognostic impact has recently been validated by an international meta-analysis [35]. They are, however, not validated for clinical decision-making.

\section{Lesions of Uncertain Malignant Potential (B3) Including Atypical Ductal Hyperplasia, Flat Epithelial Atypia, and Lobular Intraepithelial Neoplasia}

Lesions identified on core needle biopsy $(\mathrm{CNB})$ are routinely classified into 5 categories according to the B-coding system, with lesions of uncertain malignant potential being grouped into the B3 category [36]. The B3 category comprises a heterogeneous group of lesions that share an increased and clinically relevant risk of malignancy in a subsequent open biopsy. The most common lesions in the B3 category include lesions with atypical proliferation, e.g. atypical ductal hyperplasia (ADH), lobular neoplasia (LN), and flat epithelial atypia (FEA), but also papillomas because of their potential heterogeneity. The accurate pathological identification and classification of lesions with atypical proliferation is important to assess the individual risk of the patient, and to decide if the lesion should be excised. The recognition of atypical epithelial proliferation is based on the distinction of hyperplastic from neoplastic lesions, i.e. on the identification of a clonal process.

General recommendations for all types of B3 lesions identified at core biopsy include that careful attention must be paid to the pathologic-radiologic correlation for guidance of the clinical management. This is typically achieved in an interdisciplinary conference that includes the presentation of radiologic and pathologic findings. The term 'atypical ductal hyperplasia' has been defined to describe small atypical ductal lesions with insufficient criteria for a definite diagnosis of DCIS. No change was made in the general recommendation for an excisional biopsy when $\mathrm{ADH}$ is identified in $\mathrm{CNB}$ or in a vacuum-assisted biopsy specimen. FEA is a lesion with architectural features of columnar cell hyperplasia with lowgrade nuclear atypia and is considered a precursor lesion in the low-grade pathway. Management recommendations incorporate the actual risk of progression, which is low. Open biopsy must be considered when suspicious microcalcifications or a mass lesion remain radiologically detectable after core needle or vacuum-assisted biopsy [37].

With lobular intraepithelial neoplasia (LIN), several different morphologic variants of lobular carcinoma in situ (LCIS) have been described to more precisely evaluate the individual risk. Pleomorphic and florid LCIS (pLCIS and fLCIS) differ from classical LCIS (cLCIS). pLCIS was shown to behave more aggressively as compared to classical LN [38]. The distinction of pLCIS from classical LN relies on nuclear characteristics, with pLCIS having larger, more pleomorphic nuclei with obvious nucleoli and potentially showing apocrine differentiation, necrosis, and microcalcifications. fLCIS is another form of LIN with high risk, which may be frequently associated with microinvasion $[39,40]$. In the grading system of LIN (LIN 1-3), pLCIS and fLCIS are categorized as the most severe grade (LIN 3) [41].

An excisional biopsy was recommended in pLCIS or fLCIS because of an upgrade rate greater than $25 \%$ [42] or $16 \%$ [43], but results were inconclusive with lesions of lesser extent, namely atypical lobular hyperplasia. The argument against a routine follow-up biopsy is that $\mathrm{LN}$ as the most significant pathology usually is an incidental finding in an otherwise benign core biopsy, and if there is no other clinically or radiologically detectable lesion, it is unlikely that an excisional biopsy could yield anything more significant [44]. This argument has to be taken seriously, and at least all cases with LCIS and a mass lesion should be followed up by a surgical biopsy. However, because of the reported upgrade rates in fully developed LCIS, the nature of these lesions as non-obligate precursors, and the risk of missing a radiologically occult invasive cancer, an open biopsy in classical LCIS should also be considered as 
an option [45], especially if multiple lobules are involved [46-48].

\section{Ductal Carcinoma in Situ}

In addition to invasive breast cancer, the estimated annual incidence of in situ breast cancer in the USA for 2013 was 64,640 . Of these, approximately $85 \%$ will be DCIS cases. The in situ breast cancer incidence rates increased by $2.8 \%$ per year from 2005 to 2009 [49]. Due to the screening programs, breast cancer is detected at earlier stages, with the detection of non-palpable lesions and in situ carcinomas having increased up to approximately $20 \%$ of the newly diagnosed primary cases [50].

For successful BCS, the primary lesion has to be removed with a surrounding rim of healthy tissue of at least $2 \mathrm{~mm}$. The challenge is to achieve clear margins in 1 surgical session. In general, positive margins indicate a re-excision procedure. Because of insufficient intraoperative assessment of the lesion extension, the re-excision rates for DCIS reported in the majority of studies are high, ranging from 31 to $46 \%$ for DCIS only [51-54]. In contrast to invasive breast cancer there are just a very few possibilities to intraoperatively assess the specimen margin (touch prep cytology, spectroscopy) and to lower the re-operation rate. Ahmed and Douek [55] published a systematic review and meta-analysis to evaluate the impact of intraoperative US (IOUS) in comparison to wire-guided localization (WGL) in non-palpable breast cancers and DCIS. The authors concluded that, compared with WGL, IOUS reduced the involved surgical margin rates in non-palpable lesions as long as they were visible. Therefore, for invisible DCIS, IOUS cannot be recommended (LoE 2b, B, AGO +/-).

Radiotherapy to the breast in BCT reduces local recurrences by $50 \%$ but is not associated with a survival benefit. Since no subgroup of patients has been defined so far not to benefit from radiotherapy (with respect to local control), radiotherapy should be offered to all patients with BCT. RT can be omitted in selected patients with a minimal absolute risk of recurrence provided shared decision-making and careful information of the patient regarding risks and benefit is provided. This was repeatedly confirmed by a Cochrane analysis of postoperative radiotherapy for DCIS of the breast (LoE 1a, A, AGO ++) [56]. A multigene assay called DCIS Score ${ }^{\circledR}$ showed promising data to classify patients into 3 distinct risk groups (low, intermediate, high). This test may be able to verify a group of patients with low risk in which radiotherapy can be avoided in the future [57].

HER2-positive DCIS patients were included in the National Surgical Adjuvant Breast and Bowel Project (NSABP) B-43 trial, which investigates the impact of trastuzumab given concurrently with radiation therapy to radiation therapy alone. This trial is now fully recruited. Surprisingly, only $34.9 \%$ of the patients were HER2 positive $(1,969)$, a lower rate than previously reported. Only safety data have been published as yet and no grade 4 or 5 toxicity has been observed. Nevertheless, there is no indication for trastuzumab in DCIS (LoE 5, D, AGO -/-) [58]. More data from this trial will be awaited.

\section{Breast Cancer Surgery - Oncological Aspects}

Axillary lymph node involvement is a strong prognostic marker, and in the absence of reliable imaging systems to assess the nodal status, axillary lymph node dissection (ALND) remained the best staging tool. When axillary sentinel lymph node biopsy (SLNB) was introduced, a less radical surgical treatment evolved, with a remarkable reduction in postoperative morbidity. Today, axillary dissection is mainly a diagnostic procedure and improves clinical outcome only in patients with lymph node metastases. Removal of tumor-free lymph nodes increases morbidity and has no prognostic impact. Available evidence suggests that quality-assured SLNB is a reliable predictor of axillary lymph node status, with high levels of sensitivity (90-95\%), specificity (100\%), NPV (95\%), and accuracy (97\%) [59].

The systemic treatment in patients who undergo neoadjuvant therapy is generally predefined. In these patients, the histopathological response to chemotherapy (which includes response in the breast and the lymph nodes) is an important prognostic factor with significant potential to tailor future systemic and regional treatment decisions. Therefore, it would seem more reasonable to perform SLNB after NACT in order to provide this important prognostic factor.

SLNB after NACT is, however, associated with less favorable success rates (detection rate, false-negative rate) compared to SLNB in primary surgery, as shown in the SENTINA trial. This relates especially to patients, who present initially with positive lymph nodes and convert to a negative axillary status under NACT. For patients with initially negative lymph nodes, the success rates for SLNB after NACT appear more favorable, although evidence from sufficiently powered prospective trials is still lacking. Furthermore, no data regarding oncologic endpoints (disease-free survival, overall survival) are yet available for the SLN procedure after NACT.

Patients with a positive axillary finding before NACT may have a diagnostic core needle or fine-needle biopsy to confirm axillary involvement cytologically/histologically prior to initiating chemotherapy. Proven axillary lymph node involvement (needle biopsy or sentinel node biopsy) is an indication for ALND following NACT. One option is sentinel lymph node dissection after NACT, with a minimum of 3 sentinel lymph nodes (false-negative rate $<10 \%$; American College of Surgeons Oncology Group (ACOSOG) Z1071 trial) $[60,61]$.

In case of positive SLNB before NACT (fig. 1), ALND may be omitted under certain conditions (tumor $<5 \mathrm{~cm}, \mathrm{cN} 0$, 


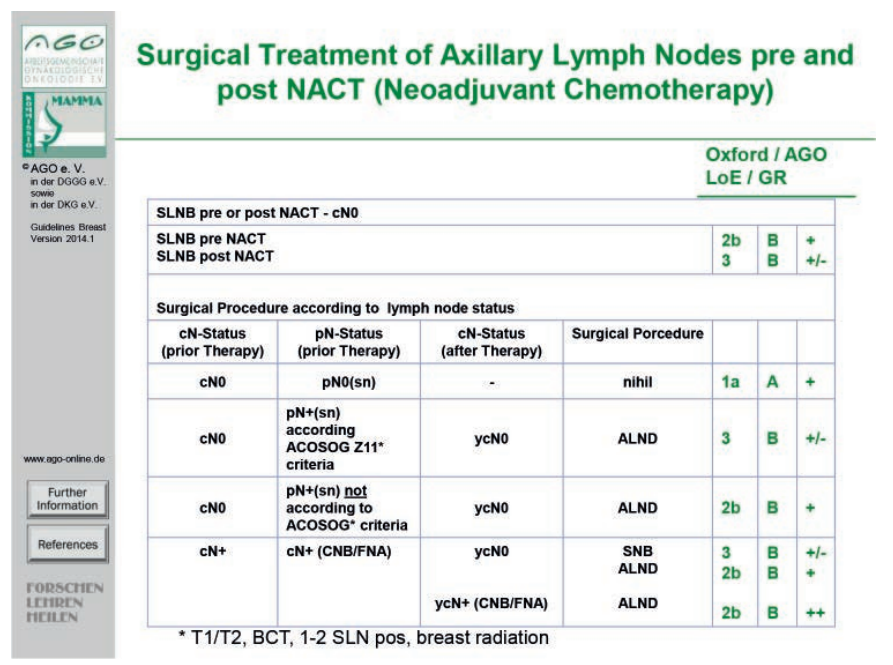

Fig. 1. Surgical treatment of axillary lymph nodes before and after NACT.

less than 3 lymph nodes affected, no extracapsular spread, planned whole breast irradiation for BCS, planned adequate systemic therapy (ACOSOG Z011 trial) following informed consent). In case of positive SLNB after NACT, prospective data to avoid axillary dissection are lacking and must be taken into consideration in planning axillary surgical procedures under neoadjuvant treatment conditions.

In conclusion, SLNB prior to NACT is a safe procedure that can spare many patients with advanced tumors an axillary dissection. SLNB after NACT is an important development that should, however, be performed within clinical trials to provide the necessary and urgently awaited data on clinical outcome.

Axillary dissection and radiotherapy are both associated with excellent regional control rates in clinically node-negative patients with a positive sentinel lymph node as shown in the AMAROS trial (LoE 1b, B, AGO +/-) [62]. Patients who received radiotherapy had significantly less arm morbidity compared to patients who underwent axillary dissection. However, many questions remain, such as the necessity of internal and supra-infra node irradiation; thus, publication of the full paper of the AMAROS trial should be awaited before radiotherapy is used routinely to replace axillary surgery in patients who require axillary dissection.

\section{Oncoplastic and Reconstructive Surgery}

Oncoplastic surgery in its original form began as combining lumpectomy or quadrantectomy with local or regional tissue rearrangement, so that the breast should be conserved and reshaped to avoid significant deformity. Oncoplastic techniques should be used in BCS in case of an expected breast volume loss > 10-20\%; a wider clear margin and a lower re-excision rate can be achieved. If BCS is not possible, breast reconstruction should be offered to the patients. The first choice is the reconstruction by implants. Skin-sparing mastectomy (SSM) or nipple-sparing mastectomy (NSM) are safe and afford a higher quality of life (LoE 2b, B, AGO ++) [63]. The preservation of the nipple-areola complex (NAC) can be performed under special conditions ( $\mathrm{LoE} 2 \mathrm{~B}, \mathrm{~B}, \mathrm{AGO}++$ ). SSM with preservation of the NAC is also feasible after mastopexy or reduction mammoplasty ( $\mathrm{LoE} \mathrm{4,} \mathrm{C,} \mathrm{AGO} \mathrm{++)} \mathrm{[64].}$

If radiotherapy is indicated, the use of implants is possible regarding oncologic safety ( $\mathrm{LoE} 2 \mathrm{a}, \mathrm{B}, \mathrm{AGO}+/-$ ). Patients should be informed in detail about higher complication rates and poorer cosmetic results.

Synthetic meshes or acellular dermal matrices (ADM) are possible options for muscle fixation in case of immediate reconstruction ( $\mathrm{LoE} \mathrm{2b}, \mathrm{C}, \mathrm{AGO}+)$. The use of ADM presents no significant increase of long-term complication rates and less capsular contracture compared to 2-stage expander/implant reconstruction without ADM (LoE 2b, C) [65]. Synthetic meshes can be used in patients undergoing reconstruction after SSM or NSM with well-preserved skin soft-tissue proportions and in patients with primary or secondary prophylactic subcutaneous mastectomy; they seem to be a helpful tool for implant stabilization in terms of lateral stabilization and fixation of the musculus pectoralis major [66].

The use of lipomodeling by autologous fat transfer is increasing, offering an additional tool to refine breast-reconstructive surgery [67]. There are reported concerns that the injection of fat may be involved in tumorigenesis by stimulating angiogenesis and cell growth and thus dormant cancer cells [68]. Lipofilling has already been performed for breast reconstruction in over 2,000 patients in published trials. Until now, there has been no report of increasing risk of local events or metastasis in the follow-up of invasive breast cancer patients [69]. By this, lipofilling can be offered and performed after mastectomy and implant-based reconstruction (LoE 2a, $\mathrm{B}, \mathrm{AGO}+)$. However, lipofilling presented an increased risk of local events in subgroups of women younger than 50 years with high-grade neoplasia and Ki-67 > 14\% [70]. This option should be considered carefully in these subgroups.

After BCS, lipofilling should only be performed on an individual basis and after detailed informed consent, due to lack of data (LoE 4, D, AGO +/-). A longer interval after diagnosis should be considered [71].

If implant reconstruction is not suitable, pedicled (LoE 3b, $\mathrm{C}, \mathrm{AGO}+$ ) or free tissue flaps $(\mathrm{LoE} 3 \mathrm{~b}, \mathrm{~B}, \mathrm{AGO}+/-)$ can be considered. Comparing both techniques, the free tissue transfer is a time- and personnel-consuming microsurgical procedure associated with a higher rate of reoperations, a higher total failure rate, and no higher patient satisfaction in multivariate analyses. In case of transverse rectus abdominis muscle (TRAM) flaps, a delayed procedure should be performed in risk patients $(\mathrm{LoE} 3 \mathrm{a}, \mathrm{B}, \mathrm{AGO}+)$. Moreover, the ipsilateral pedicled TRAM is recommended ( $\mathrm{LoE} 3 \mathrm{~b}, \mathrm{~A}, \mathrm{AGO}+)$.

Risk-reducing bilateral mastectomy in healthy women (RRBM - former prophylactic mastectomy) can achieve a 
reduction in breast cancer incidence ( $\mathrm{LoE} 1 \mathrm{~b}, \mathrm{~A}, \mathrm{AGO}++)$. It may be offered to patients with a BRCA1/2 mutation (LoE $2 \mathrm{a}$, $\mathrm{B}, \mathrm{AGO}+$ ) or in high-risk women (i.e. with a lifetime risk $\geq$ $30 \%$ or a heterozygote risk $\geq 20 \%$ ) ( $\operatorname{LoE} 3 \mathrm{a}, \mathrm{C}, \mathrm{AGO}+/-$ ). Possible techniques are simple mastectomy, SSM, and NSM (NAC sparing) (LoE 2b, C, AGO +). Actual data present a high need for education of physicians - especially in Germany - regarding the indications, possibilities, and advantages of RRBM (LoE 1b, A, AGO ++) [72].

\section{Adjuvant Endocrine Therapy}

Adjuvant endocrine therapy represents the most important therapeutic option in the treatment of early breast cancer. As endocrine therapy represents a targeted treatment option, a basic requirement is the proof of endocrine sensitivity (ES). ES by definition requires that at least $1 \%$ of the tumor cells should stain positive for either estrogen or progesterone receptors. In premenopausal women, 5-year treatment with tamoxifen is the standard regimen. In special cases (e.g. marked formation of ovarian cysts), suppression of the ovaries with gonadotropin-releasing hormone $(\mathrm{GnRH})$ analogs may be useful. In postmenopausal patients, there are 3 treatment options available for the duration of 5 years:

- 5 years of tamoxifen,

- 5 years of aromatase inhibition or

- a sequence therapy with 2 years of tamoxifen followed by an aromatase inhibitor for 3 years or 2 years with an aromatase inhibitor followed by 3 years of tamoxifen. This treatment is associated with the best therapeutic index for these patients.

When lobular breast cancer occurs, upfront therapy using an aromatase inhibitor might be beneficial. However, these data have only been presented at conferences and have not yet been fully published. In recent years, the importance of extended adjuvant therapy has been increasingly discussed. The ATLAS trial [73] and the aTTom [74] study referred to the benefit of extended endocrine therapy with tamoxifen beyond 5 years (LoE 1a, A, AGO ++). Both studies reported a reduction in breast cancer-related mortality of $1.9 \%$. However, this reduction in tumor-related mortality was associated with an increase in treatment-related mortality, when considered over the entire study. This increase was equal to the breast cancer-related mortality reduction.

The reduction in breast cancer-related mortality was induced by the reduction of intramammary recurrence and was attributed to the reduction of secondary cancers. It is therefore a tertiary preventive approach, by nature. Thus, this extended adjuvant therapy should be offered only to women who have an increased locoregional risk of recurrence or an increased risk of a second breast cancer.

For premenopausal women, in these cases, 10-year tamoxifen therapy is an option to reduce their overall risks. In post- menopausal women, after 5 years of tamoxifen, there are 2 options for extended adjuvant therapy (EAT) proposing extension with another 5 years of tamoxifen or letrozol as demonstrated in the MA.17 trial [75]. Treatment with these drugs is associated with a lower risk of venous thromboembolism (VTE) and secondary cancers like endometrium cancer. The remarkable efficacy of this option could be demonstrated 10 years ago in the Canadian MA.17 trial ( $L$ E 2b, B, $\mathrm{AGO}+$ ) [75]. At present there are no clear data as to which of the 2 options of EAT might be the treatment of choice. It is of importance to understand that EAT is not recommended in the first 5 years after a sequence treatment.

\section{Adjuvant Cytotoxic and Targeted Therapy}

Systemic treatment is generally recommended according to the specific subtypes of breast cancer. In patients with hormone receptor-positive, HER2-negative and low-risk biology, endocrine therapy without chemotherapy is recommended.

In patients with hormone receptor-positive, HER2-negative and high-risk situation, conventionally dosed anthracycline/taxane-based chemotherapy is the first choice, followed by endocrine therapy. In patients with HER2-overexpressing tumors, sequential anthracycline/taxane-based regimens with concurrent taxane and trastuzumab are recommended; another choice is an anthracycline-free, carboplatincontaining regimen. In patients with triple-negative breast cancer (TNBC), conventionally dosed anthracycline/taxanebased chemotherapy is the first choice.

We agreed on considering the neoadjuvant approach in all patients with an indication for adjuvant chemotherapy, mainly based on tumor biology.

Based on data from the Italian Grupo Italiano Mamella (GIM) study presented by Cognetti et al. [76] at the San Antonio Breast Cancer Symposium (SABCS) 2013, epirubicin/cyclophosphamide (EC) followed by paclitaxel is equally effective to EC plus 5-fluorouracil (FEC) followed by paclitaxel. Therefore, our new guideline recommendation is EC without the addition of 5-fluorouracil. The best combinations are either EC 90/600 q3w $\times 4$ followed by paclitaxel $80 \mathrm{qw} \times 12$ or docetaxel 75, adriamycin 50, cyclophosphamide $600 \mathrm{q} 3 \mathrm{w} \times 6$ (TAC). Sequential regimens like $4 \times$ adriamycin/cyclophosphamide $(\mathrm{AC}) \mathrm{q} 3 \mathrm{w}$ followed by docetaxel $100 \mathrm{q} 3 \mathrm{w} \times 4$ or FEC $\mathrm{q} 3 \mathrm{w} \times 3$ followed by docetaxel $\mathrm{q} 3 \mathrm{w} \times 3$ are also feasible.

For dose-dense regimens, additional knowledge has accumulated in the year 2014:

- The Italian GIM study presented by Cognetti et al. [76] has shown that EC 90/600 q2w with pegfilgrastim followed by paclitaxel $175 \mathrm{q} 2 \mathrm{w}$ is superior to the same regimen applied at 3-weekly intervals.

- The NSABP B38 trial published by Swain et al. [77] has shown that 6 cycles of TAC is equally effective to dosedense EC followed by paclitaxel $\mathrm{q} 2 \mathrm{w}$. 
- The Southwest Oncology Group (SWOG) S0221 study presented by Budd et al. [78] at the ASCO meeting 2013 has shown that paclitaxel $175 \mathrm{q} 2 \mathrm{w} \times 6$ is equally effective to paclitaxel $80 \mathrm{mg} / \mathrm{m}^{2}$ weekly $\times 12$.

- Therefore, the general consideration for anthracycline/ taxane treatment in the adjuvant setting would be optimally $4 \times$ EC followed by $12 \times$ paclitaxel qw [79].

- At the moment, it cannot be decided whether this regimen or the German dose-dense, dose-intensified regimen ETC (epirubicin, paclitaxel, cyclophosphamide) are equally effective [80].

- The answer to this question will possibly be given by the mature results of the German Adjuvant Intergroup Nodepositive (GAIN, ETC vs. EC followed by paclitaxel + capecitabine) study.

\section{Adjuvant Treatment for Patients with \\ HER2-Positive Tumors}

For the adjuvant therapy of patients with HER2-overexpressing tumors, trastuzumab is recommended for the duration of 1 year in patients with node-positive disease and also in patients with node-negative disease if the tumor has a diameter of more than $10 \mathrm{~mm}$ [81-83].

In patients with a tumor diameter between 5 and $10 \mathrm{~mm}$, we also recommend the use of chemotherapy plus trastuzumab. In patients with a tumor diameter of $<5 \mathrm{~mm}$, an individual discussion with the patient should be performed in order to decide on whether to treat with chemotherapy and trastuzumab. In the study presented by Tolaney et al. [84] at the SABCS 2013, chemotherapy was performed with 12 weeks of weekly paclitaxel with the addition of trastuzumab followed by trastuzumab in patients with tumors of less than $3 \mathrm{~cm}$ in diameter and no involved axillary nodes. The 5-year outcome of these patients was excellent; we therefore recommend such a regimen in individual cases based on discussions with the patients [84]. This is a highly feasible regimen in postmenopausal patients with additional comorbidities and pT1 N0 situation.

\section{Neoadjuvant Chemotherapy}

NACT is indicated in every case with an indication for adjuvant chemotherapy ( $\mathrm{LoE} 1 \mathrm{~b}, \mathrm{~A}, \mathrm{AGO}+)$. In particular, in patient subgroups where a pCR is associated with improved survival, such as in triple-negative and HER2-positive cancers, NACT (plus targeted therapy) should be the preferred therapeutic approach $(\mathrm{AGO}+)$. Independent of the BRCA1/2 status, a platinum salt-containing regimen should be considered (LoE 2b, B, AGO +) in TNBC based on data from several phase II randomized trials (e.g. GeparSixto, CALGB 40603).

Response-guided treatment has been shown to be beneficial within the GeparTrio trial. Consequently, in case of response after 2 cycles of DAC in hormone receptor-positive breast cancer, a total of 8 instead of 6 cycles of DAC may be considered $(\mathrm{LoE} 2 \mathrm{~b}, \mathrm{C}, \mathrm{AGO}+)$. In the case of no response after 2 cycles of DAC, continuation of neoadjuvant systemic therapy with a non-cross-resistant regimen, such as $4 \times$ vinorelbine/capecitabine (NX) may be beneficial ( $\mathrm{LoE} 2 \mathrm{~b}, \mathrm{~B}$, $\mathrm{AGO}+$ ). This can be an option in individual cases but cannot be considered a routine approach.

With respect to endocrine neoadjuvant therapy, in exceptional situations endocrine treatment with luteinizing hormone-releasing hormone (LHRH) plus aromatase inhibitor may be considered for premenopausal women (LoE 1b, C, $\mathrm{AGO}+/-$ ).

New predictive factors, such as tumor cell infiltration/ lymphocyte-predominant breast cancer or phosphatidylinositol-4,5-bisphosphate 3-kinase catalytic subunit alpha (PIK3CA) mutation in the tumor, are promising tools but not yet applicable in the routine clinical setting (LoE II, B, $\mathrm{AGO}+$ ).

The indications for mastectomy after NACT remain unchanged: positive margins after repeated excisions (LoE 3b, $\mathrm{C}, \mathrm{AGO}++)$, radiotherapy not feasible ( $\mathrm{LoE} 5, \mathrm{D}, \mathrm{AGO}++)$, and inflammatory breast cancer in the case of only clinical complete response ( $\mathrm{LoE} 2 \mathrm{~b}, \mathrm{C}, \mathrm{AGO}+)$. In inflammatory breast cancer with pCR after NACT, BCS may be discussed with the patient ( $\mathrm{AGO}+/-)$ as an individual option, with mentioning of the scarce database for such an approach.

Similarly, large tumors (cT4a-c) are only a relative indication for mastectomy after NACT (AGO +/-). Multicentric lesions should be exactly defined (bicentric, tricentric), and the remaining tumor load after NACT must be set in relation to the breast size $(\mathrm{AGO}+/-)$.

Postneoadjuvant concepts are currently investigated in clinical trials. There is no indication for further chemotherapy in case of no pCR.

\section{Adjuvant Radiotherapy}

Due to new results published 2013 from meta-analyses and prospective randomized clinical trials regarding radiooncological aspects, the AGO Breast Committee has reevaluated some current issues relevant to clinical practice.

There is no subgroup - including elderly patients - without any benefit of normofractionated (nf) radiotherapy (RT) after BCS of DCIS and invasive cancer in terms of significant increase of local/locoregional tumor control resulting in a ++ recommendation of the AGO. However, for elderly patients there is no impact of postoperative radiotherapy on breast cancer-related survival. There are more severe cardiovascular events after radiotherapy of the breast [56, 85-89].

Regarding the role of (accelerated) partial breast irradiation/intraoperative (electron) radiotherapy (IO(E)RT) after $\mathrm{BCS}$ as sole radiotherapy modality, the prospective rand- 
omized TARGIT A trial (comparing IORT with external beam radiotherapy (EBRT)) could show no significant increase of local recurrence with risk-adapted intraoperative prepathology radiotherapy with $50 \mathrm{kV}$. Risk-adapted prepathology IORT means partial breast radiation at the time of first surgery plus an additional EBRT or, in case of unexpected risk factors like postoperative lymphangiosis carcinomatosa ( $\mathrm{pL} 1)$, an additional EBRT. The inclusion criteria were an age older than 45 years (in Germany, older than 50 years), no lobular invasive cancer, no extensive intraductal component (EIC, defined as the presence of intraductal carcinoma both in the invasive tumor and in adjacent breast tissue that comprised $>25 \%$ of the tumor), cancer $<2 \mathrm{~cm}$ (Germany), and hormone receptor-positive cancer. The other randomized trial compares IOERT with EBRT. The patients were not selected. The local recurrence rate was significantly higher with IOERT, but very low in both study arms [90-96].

With regard to the duration of radiotherapy by appropriately dosed hf schedules (i.e. single fractions of $2.66 \mathrm{~Gy}$, restriction of total dose to 39-41.6 Gy), the recently published 10-year follow-up data of the UK START A/B and the Canadian trials were considered to indicate safety and effectivity. Consequently, the British National Institute for Health and Clinical Excellence (NICE) confirmed this approach as the new standard and recommended UK START B-hf-RT in its guidelines [97-100]. Out of all these trials dealing with hf radiotherapy schedules, only the UK START B trial could also demonstrate a minimal but statistically significant overall survival benefit [97]. Based on these results, the AGO Breast Committee members now opted $++(\operatorname{LoE} 1 \mathrm{a}, \mathrm{A}, \mathrm{AGO}++)$ in favor of hf as compared to $+(\operatorname{LoE} 1 \mathrm{a}, \mathrm{A}, \mathrm{AGO}+)$ for $\mathrm{nf}$ schedules. This vote disagrees with the recommendations regarding hf concepts from the updated German Society of Radiation Oncology (DEGRO) practical guidelines 2013/14 [101].

A tumor bed boost radiotherapy as part of a whole-breast irradiation (WBI) concept is as well as feasibility in hf regimens. In addition to WBI it reduces local recurrence in all age groups and should therefore be offered to patients who appear biologically and mentally fit enough to experience the benefit of improved local control. Results of ongoing clinical trials evaluating different boost concepts (e.g., simultaneously integrated boost (SIB), anticipated boost applying IO(E)RT prior to WBI) and within $\mathrm{nf}$ and hf concepts are expected.

A meta-analysis and further data from randomized controlled trials have improved the evidence levels indicating postmastectomy irradiation (PMRT) [102-105]. PMRT is beneficial for all patients with 'high-risk' criteria in terms of both locoregional tumor control and overall survival. However, it is still a matter of debate if this is most likely also true for patients with 'intermediate-risk' criteria (i.e. relapse risk (RR) 10-20\%; T1-2 and $\mathrm{N}+(1-3)$, G3, vascular invasion, lobular subtype; > T2 N0). In pN0 patients, recommendation for PMRT depends on the number of risk factors for loco- regional relapse. For the pN0 subgroup bearing more than 1 risk factor, PMRT is considered to complete treatment in these patients. Now, the recent meta-analysis of Li et al. [103] covering more than 3,400 patients as well as further retrospective cohort analyses confirmed statistically significant beneficial effects of PMRT even in patients with 1-3 positive lymph nodes in terms of locoregional tumor control. Therefore, PMRT should be recommended for patients with 1-3 positive lymph nodes (LoE 1a, A, AGO +) [102-104, 106]. At present, it is unclear which subgroups with indication for PMRT (high risk, pN0 or intermediate pN1 (1-3 lymph nodes)) would benefit from an inclusion of the regional lymph nodes into the radiotherapy target. Better local control through modern systemic treatments has to be taken into consideration. Recently published studies were in their time conducted without modern therapies as aromatase inhibitors and anti-HER2 therapies were not yet available.

\section{Complementary Treatment}

Generally, the AGO recommendations tend to remain unchanged. Trends seen in recent previous years are followed and some vague recommendations are being slightly consolidated. It has become clear that the greatest danger of complementary and alternative medicine (CAM) is in omitting conventional therapy, specifically systemic therapy and surgery. This could be clearly demonstrated by Saquib et al. [107]. The purpose of their study was to assess whether CAM approaches affected breast cancer prognosis in those patients who did not receive systemic therapy. They performed a secondary data analysis of baseline/survey data from the Women's Healthy Eating and Living (WHEL) study including 2,562 breast cancer survivors. The mean follow-up approached 7.3 years. Those women who did not receive any systemic treatment had a higher risk for time to additional breast cancer events (hazard ratio $(\mathrm{HR})=1.9,95 \%$ confidence interval (CI) 1.32-2.73) and for all-cause mortality (HR $=1.7,95 \%$ CI 1.06-2.73) compared to those who had received systemic treatment. Among 177 women who did not receive systemic treatment, CAM use was not significantly related with additional breast cancer events. The use of dietary supplements or CAM therapies did not change this risk. This indicates that complementary and alternative therapies did not alter the outcome of breast cancer and should not be used in place of standard treatment.

New data from randomized controlled trials enhance the impact of mind-body medicine such as yoga, chi-gong, tai-chi, and hypnosis for improving sleep, fatigue, quality of life, and physical performance. Viscum album preparations improve quality of life and side effects in patients after chemotherapy. The 5-year results of a placebo-controlled trial of coenzyme Q10 to relieve self-reported treatment-related fatigue were negative. 
Quite a bit of new information has accumulated on immunotherapeutic approaches. Most modalities do not boast high evidence levels and rather represent early exploratory trials, however indicating that this enigmatic field holds future promise. The danger in these methods is that patients are prone to fraudulent offers from 'immunotherapists' and 'healers' claiming that boosting the immune system will cure early and progressive disease, as the black-box promise of immunotherapy is attractive and seemingly self-explanatory for distraught patients.

Recently, the discovery that chronic inflammation in the tumor microenvironment promotes tumor growth and survival during different stages of breast cancer development has led to the development of novel immunotherapies. Several immunotherapeutic strategies have been studied both preclinically and clinically and have already been shown to enhance the efficacy of conventional treatment modalities. Therefore, therapies targeting the immune system may represent a promising next-generation approach for the treatment of breast cancers.

\section{Dendritic Cell Intradermal Vaccination}

In a recent paper from China [108], dendritic cell (DC) vaccines were generated from CD14+ precursors pulsed with autologous tumor lysates. DCs were matured with defined factors that induced surface marker and cytokine production. Individuals were immunized intradermally 4 times. Overall survival and disease progression rates were compared with those of contemporaneous patients who did not receive DC vaccines. There was no difference in overall survival between the patients with and without DC vaccine administration. The 3-year progression-free survival was significantly prolonged: $76.9 \%$ versus $31.0 \%$ (with vs. without DC vaccine, $\mathrm{p}<0.05$ ). The authors concluded that their findings strongly suggest that tumor lysate-pulsed DCs provide a standardized and widely applicable source of breast cancer antigens that are very effective in evoking anti-breast cancer immune responses.

Recently, quite a number of preliminary clinical trials have been evaluated and published reporting on immunotherapeu- tic interventions yielding promising early results. In this context, Montero et al. [109] reported on the addition of NOV002 (a formulation of disodium glutathione disulfide) to chemotherapy, which has been shown to increase antitumor efficacy in animal models and some early-phase oncology trials. Concurrent NOV-002 resulted in a pCR rate of $38 \%$ for $\mathrm{AC} \rightarrow \mathrm{T}$ ( $\mathrm{T}=$ docetaxel) chemotherapy, which is higher than previously reported rates, e.g. in the $\mathrm{B} 27$ or the GeparDuo trials.

This and other early indications of efficacy do not at all allow general recommendation; so, all these approaches can only be recommended within the settings of properly controlled trials.

However, one new piece of information could be ready for clinical application in difficult situations without better and more promising alternatives: the tentative treatment of skin metastases with the immunomodulator imiquimod. A prospective case series was published by Adams et al. [110], evaluating the local tumor response rate of breast cancer skin metastases treated with topical imiquimod, applied 5 days/ week for 8 weeks. 10 patients were enrolled. 2 patients achieved a partial response. The responders showed histologic tumor regression with evidence of an immune-mediated response, shown by changes in the tumor lymphocytic infiltrate and locally produced cytokines.

\section{Disclosure Statement}

C.L. has received consultant and speaker honoraria from Amgen, Celgene, Genomic Health, GSK, Novartis, Pierre-Fabre, Roche, and TEVA. M.T. has received consultant and speaker honoraria from AstraZeneca, Amgen, Celgene, Genomic Health, GSK, Novartis, Pierre-Fabre, Serag-Wiessner, Roche, and Sysmex Europe. V.H. has received consultant and speaker honoraria from Novartis and Roche. F.S. has received consultant and speaker honoraria from AstraZeneca, Amgen, Celgene, Novartis, Roche Pharma, and medtronic. The authors have no relevant affiliation or financial involvement with any organization or entity with financial interest in or financial conflict with the subject matter or materials discussed in the manuscript apart from the disclosed.

\section{References}

1 Centre for Evidence Based Medicine: Oxford Level of Evidence, LoE. www.cebm.net.

2 www.ago-online.de/de/fuer-mediziner/leitlinienempfehlungen/mamma/.

3 Ritte R, Tikk K, Lukanova A, et al.: Reproductive factors and risk of hormone receptor positive and negative breast cancer: a cohort study. BMC Cancer 2013;13:584.

4 Gonzalez CA: Diet and cancer prevention: contributions from the European Prospective Investigation into Cancer and Nutrition (EPIC) study. Eur J Cancer 2010;46:2555-2562.

5 Chlebowski RT, McTiernan A, Wactawski-Wende J, et al.: Diabetes, metformin and breast cancer in postmenopausal women. J Clin Oncol 2012;30: 2844-2852.
6 McDonald JA, Goyal A, Terry MB: Alcohol intake and breast cancer risk: weighing the overall evidence. Curr Breast Cancer Rep 2013;5(3).

7 Moorman PG, Havrilesky LJ, Gierisch JM: Oral contraceptives and risk of ovarian cancer and breast cancer among high-risk women: a systematic review and meta-analysis. J Clin Oncol 2013;31: 4188-4198.

8 Plon SE, Eccles DM, Easton D, et al.: Sequence variant classification and reporting: recommendations for improving the interpretation of cancer susceptibility genetic test results. Hum Mutat 2008; 29:1282-1291.

9 Gaudet MM, Kirchhoff T, Green T, et al.: Common genetic variants and modification of penetrance of BRCA2-associated breast cancer. PLoS Genet 2010;6:e1001183.
10 Couch FJ, Wang X, McGuffog L, et al.: Genomewide association study in BRCA1 mutation carriers identifies novel loci associated with breast and ovarian cancer risk. PLoS Genet 2013;9:e1003212.

11 Mavaddat N, Barrowdale D, Andrulis IL, et al.: Pathology of breast and ovarian cancers among BRCA1 and BRCA2 mutation carriers: results from the Consortium of Investigators of Modifiers of BRCA1/2 (CIMBA). Cancer Epidemiol Biomarkers Prev 2012;21:134-147.

12 Garcia-Closas M, Chanock S: Genetic susceptibility loci for breast cancer by estrogen receptor status. Clin Cancer Res 2008;14:8000-8009.

13 Weischer M, Nordestgaard BG, Pharoah P, et al.: CHEK2*1100delC heterozygosity in women with breast cancer associated with early death, breast cancer-specific death, and increased risk of a 
second breast cancer. J Clin Oncol 2012;30:43084316.

14 Gartlehner G, Thaler K, Chapman A, et al.: Mammography in combination with breast ultrasonography versus mammography for breast cancer screening in women at average risk. Cochrane Database Syst Rev 2013;(4):CD009632.

15 de Bock GH, Vermeulen KM, Jansen L, et al. Which screening strategy should be offered to women with BRCA1 or BRCA2 mutations? A simulation of comparative cost-effectiveness. Br J Cancer 2013;108:1579-1586.

16 Bosse K, Graeser M, Goßmann A, et al.: Supplemental screening ultrasound increases cancer detection yield in BRCA1 and BRCA2 mutation carriers. Arch Gynecol Obstet 2014;289:663-670.

17 Fischer T, Sack I, Thomas A: Characterization of focal breast lesions by means of elastography. Röfo 2013;185:816-823.

18 Houssami N, Turner R, Macaskill P, et al.: An individual person data meta-analysis of preoperative magnetic resonance imaging and breast cancer recurrence. J Clin Oncol 2014;32:392-401.

19 Houssami N, Turner R, Morrow M: Preoperative magnetic resonance imaging in breast cancer: meta-analysis of surgical outcomes. Ann Surg 2013; 257:249-255.

20 Krekel NM, Haloua MH, Lopes Cardozo AM, et al.: Intraoperative ultrasound guidance for palpable breast cancer excision (COBALT trial): a multicentre, randomised controlled trial. Lancet Oncol 2013;14:48-54.

21 Pan H, Wu N, Ding H, et al.: Intraoperative ultrasound guidance is associated with clear lumpectomy margins for breast cancer: a systematic review and meta-analysis. PLoS One 2013;8:e74028.

22 Houssami N, Diepstraten SC, Cody HS 3rd, et al.: Clinical utility of ultrasound-needle biopsy for preoperative staging of the axilla in invasive breast cancer. Anticancer Res 2014:34:1087-1097.

23 De Los Santos J, Cantor A, Amos KD, et al. Magnetic resonance imaging as a predictor of pathologic response in patients treated with neoadjuvant systemic treatment for operable breast cancer. Translational Breast Cancer Research Consortium Trial 017. Cancer 2013;15:1776-1783.

24 Lobbes MBI, Prevos R, Smidt M, et al.: The role of magnetic resonance imaging in assessing residual disease and pathologic complete response in breast cancer patients receiving neoadjuvant chemotherapy: a systematic review. Insights Imaging 2013; 4:163-175.

25 Marinovich ML, Houssami N, Macaskill P, et al.: Meta-analysis of magnetic resonance imaging in detecting residual breast cancer after neoadjuvant therapy. J Natl Cancer Inst 2013;105:321-333.

26 Marinovich ML, Sardanelli F, Ciatto S, et al.: Early prediction of pathologic response to neoadjuvan therapy in breast cancer: systematic review of accuracy of MRI. Breast 2012;21:669-677.

27 Richard R, Thomassin I, Chapellier M, et al.: Diffusion-weighted MRI in pretreatment prediction of response to neoadjuvant chemotherapy in patients with breast cancer. Eur Radiol 2013;23:2420-2431.

28 Wolff AC, Hammond ME, Hicks DG, et al.; American Society of Clinical Oncology; College of American Pathologists: Recommendations for human epidermal growth factor receptor 2 testing in breast cancer: American Society of Clinical Oncology/College of American Pathologists clinical practice guideline update. J Clin Oncol 2013;31:3997-4013.

29 Rakha EA, Starczynski J, Lee AH, et al.: The updated ASCO/CAP guideline recommendations for HER2 testing in the management of invasive breast cancer: a critical review of their implications for routine practice. Histopathology 2014;64:609-615.

30 Simon RM, Paik S, Hayes DF: Use of archived specimens in evaluation of prognostic and predictive biomarkers. J Natl Cancer Inst 2009;101:14461452.

31 Harbeck N, Schmitt M, Meisner C, et al.; ChemoN0 Study Group: Ten-year analysis of the prospective multicentre Chemo-N0 trial validates American Society of Clinical Oncology (ASCO)-recommended biomarkers uPA and PAI-1 for therapy decision making in node-negative breast cancer patients. Eur J Cancer 2013;49:1825-1835.

32 Gnant M, Filipits M, Greil R, et al.; Austrian Breast and Colorectal Cancer Study Group: Predicting distant recurrence in receptor-positive breast cancer patients with limited clinicopathological risk: using the PAM50 risk of recurrence score in 1478 postmenopausal patients of the ABCSG-8 trial treated with adjuvant endocrine therapy alone. Ann Oncol 2014:25:339-345.

33 Sestak I, Cuzick J, Dowsett M, et al.: Prediction of late distant recurrence after 5 years of endocrine treatment: a combined analysis of 2485 patients from the ABCSG- 8 and transATAC studies using the PAM50 risk of recurrence (ROR) score. SABCS 2013; abstr S6-04.

34 Dubsky P, Brase JC, Jakesz R, et al.; Austrian Breast and Colorectal Cancer Study Group (ABCSG): The EndoPredict score provides prognostic information on late distant metastases in ER+/HER2- breast cancer patients. Br J Cancer 2013;109:2959-2964.

35 Bidard F-C, Peeters D, Fehm T, et al.: Pooled analysis of circulating tumor cells in metastatic breast cancer: findings from 1944 individual patients data. SABCS 2013; abstr

36 Perry N, Broeders M, de Wolf C, et al. (eds): European Guidelines for Quality Assurance in Breast Cancer Screening and Diagnosis, ed 4. Luxembourg, European Commission, Office for Official Publications of the European Communities, 2006.

37 Ceugnart L, Doualliez V, Chauvet MP, et al.: Pure flat epithelial atypia: is there a place for routine surgery? Diagn Interv Imaging 2013;94:861-869.

38 Chivukula M, Haynik DM, Brufsky A, et al.: Pleomorphic lobular carcinoma in situ (pLCIS) on breast core needle biopsies: clinical significance and immunoprofile. Am J Surg Pathol2008;32: 1721-1726.

39 Shin SJ, Lal A, De Vries S, et al.: Florid lobular carcinoma in situ: molecular profiling and comparison to classic lobular carcinoma in situ and pleomorphic lobular carcinoma in situ. Hum Pathol 2013;44:1998-2009.

40 Ross DS, Hoda SA: Microinvasive (T1mic) lobular carcinoma of the breast: clinicopathologic profile of 16 cases. Am J Surg Pathol 2011:35:750-756.

41 Bratthauer GL, Tavassoli FA: Lobular intraepithelial neoplasia: previously unexplored aspects assessed in 775 cases and their clinical implications. Virchows Arch 2002;440:134-138.

42 Shin SJ, Rosen PP: Excisional biopsy should be performed if lobular carcinoma in situ is seen on needle core biopsy. Arch Pathol Lab Med 2002; 126:697-701.

43 Elsheikh TM, Silverman JF: Follow-up surgical excision is indicated when breast core needle biopsies show atypical lobular hyperplasia or lobular carcinoma in situ: a correlative study of 33 patients with review of the literature. Am J Surg Pathol 2005; 29:534-543.

44 Nagi C: Lobular neoplasia on core needle biopsy does not require excision. Cancer 2008;112:2152-2158.
45 Sohn V: Lobular neoplasia: is surgical excision warranted? Am Surg 2008;74:172-177.

46 Cangiarella J: Is surgical excision necessary for the management of atypical lobular hyperplasia and lobular carcinoma in situ diagnosed on core biopsy? Arch Pathol Lab Med 2008;132:979-983.

47 Pinder S: Lobular in situ neoplasia and columnar cell lesions: diagnosis in breast core biopsies and implications for management. Pathology 2007;39: 208-216.

48 Brem R: Lobular neoplasia at percutaneous breast biopsy: variables associated with carcinoma at surgical excision. AJR Am J Roentgenol 2008;190:637-641.

49 www.cancer.org/acs/groups/content/ @epidemiologysurveilance/documents/ document/acspc-036845.pdf.

50 Veronesi U, Luini A, Botteri E, et al.: Nonpalpable breast carcinomas: long-term evaluation of 1,258 cases. Oncologist 2010;15:1248-1252.

51 Morrow M, Jagsi R, Alderman AK, et al.: Surgeon recommendations and receipt of mastectomy for treatment of breast cancer. JAMA 2009;302: 1551-1556.

52 Meijnen P, Oldenburg HS, Peterse JL, et al.: Clinical outcome after selective treatment of patients diagnosed with ductal carcinoma in situ of the breast. Ann Surg Oncol 2008;15:235-243.

53 Dillon MF, Mc Dermott EW, O'Doherty A, et al.: Factors affecting successful breast conservation for ductal carcinoma in situ. Ann Surg Oncol 2007; 14:1618-1628.

54 Dillon MF, Maguire AA, McDermott EW, et al. Needle core biopsy characteristics identify patients at risk of compromised margins in breast conservation surgery. Mod Pathol 2008;21:39-45.

55 Ahmed M, Douek M: Intra-operative ultrasound versus wire-guided localization in the surgical management of non-palpable breast cancers: systematic review and meta-analysis. Breast Cancer Res Treat 2013;140:435-446.

56 Goodwin A, Parker S, Ghersi D, Wilcken N Post-operative radiotherapy for ductal carcinoma in situ of the breast. Cochrane Database Syst Rev 2013;(11):CD000563.

57 Solin LJ, Gray R, Baehner FL, et al.: A multigene expression assay to predict local recurrence risk for ductal carcinoma in situ of the breast. J Natl Cancer Inst 2013;105:701-710.

58 Siziopikou KP, Anderson SJ, Cobleigh MA, et al. Preliminary results of centralized HER2 testing in ductal carcinoma in situ (DCIS): NSABP B-43. Breast Cancer Res Treat 2013;142:415-421.

59 Veronesi U, Viale G, Paganelli G, et al.: Sentinel lymph node biopsy in breast cancer: ten-year results of a randomized controlled study. Ann Surg 2010;251:595-600.

60 Boughey JC, Suman VJ, Mittendorf EA, et al.: Sentinel lymph node surgery after neoadjuvant chemotherapy in patients with node-positive breast cancer: the ACOSOG Z1071 (Alliance) clinical trial. JAMA 2013;310:1455-1461.

61 Kuehn T, Bauerfeind I, Fehm T, et al.: Sentinellymph-node biopsy in patients with breast cancer before and after neoadjuvant chemotherapy (SENTINA): a prospective, multicentre cohort study. Lancet Oncol 2013;14:609-618

62 Rutgers E, Donker M, Straver ME, et al.: Radiotherapy or surgery of the axilla after a positive sentinel node in breast cancer patients: final analysis of the EORTC AMAROS trial (10981/22023). J Clin Oncol 2013;31(suppl):abstr LBA 1001.

63 Agrawal A, Sibbering DM, Courtney CA: Skin sparing mastectomy and immediate breast reconstruction: a review. Eur J Surg Oncol 2013;39:320-328. 
64 Vaughn CJ, Peled AW, Esserman LJ, Foster RD: Feasibility of performing total skin-sparing mastectomy in patients with prior circumareolar mastopexy or reduction mammoplasty incisions. Ann Plast Surg 2013, Jun 19 [Epub ahead of print].

65 Hanna KR, DeGeorge BR Jr, Mericli AF, et al.: Comparison study of two types of expander-based breast reconstruction: acellular dermal matrixassisted versus total submuscular placement. Ann Plast Surg 2013;70:10-15.

66 Dieterich M, Reimer T, Dieterich H, et al.: A short-term follow-up of implant based breast reconstruction using a titanium-coated polypropylene mesh (TiLoop $\left({ }^{\circledR}\right)$ Bra). Eur J Surg Oncol 2012; 38:1225-1230.

67 Bonomi R, Betal D, Rapisarda IF, et al.: Role of lipomodelling in improving aesthetic outcomes in patients undergoing immediate and delayed reconstructive breast surgery. Eur J Surg Oncol 2013 39:1039-1045.

68 Lohsiriwat V, Curigliano G, Rietjens M, et al.: Autologous fat transplantation in patients with breast cancer: 'silencing' or 'fuelling' cancer recurrence? Breast 2011;20:351-357.

69 Rigotti G, Marchi A, Stringhini P, et al.: Determining the oncological risk of autologous lipoaspirate grafting for post-mastectomy breast reconstruction. Ann Plast Surg 2010;34:475-480.

70 Petit JY, Rietjens M, Botteri E, et al.: Evaluation of fat grafting safety in patients with intra epithelia neoplasia: a matched cohort study. Ann Oncol 2013;24:1479-1484.

71 Krumboeck A, Giovanoli P, Plock JA: Fat grafting and stem cell enhanced fat grafting to the breast under oncological aspects - recommendations for patient selection. Breast 2013;22:579-584.

72 Den Heijer M, van Asperen CJ, Harris H, et al.: International variation in physicians' attitudes towards prophylactic mastectomy - comparison between France, Germany, The Netherlands and the United Kingdom. Eur J Cancer 2013;49: 2798-2805.

73 Davies C, Pan H, Godwin J, et al.: Long-term effects of continuing adjuvant tamoxifen to 10 years versus stopping at 5 years after diagnosis of oestrogen receptor-positive breast cancer: ATLAS, a randomised trial. Lancet 2013;381:805-816.

74 Gray RG, Handley K, Bowden SJ, et al.: aTTom: Long-term effects of continuing adjuvant tamoxifen to 10 years versus stopping at 5 years in 6,953 women with early breast cancer. ASCO 2013; abstr.

75 Goss PE, Ingle JN, Martino S, et al.: A randomized trial of letrozole in postmenopausal women after five years of tamoxifen therapy for early-stage breast cancer. N Engl J Med 2003:349:1793-1802.

76 Cognetti F, Bruzzi P, De Placido S, et al.: Epirubicin and cyclophosphamide (EC) followed by paclitaxel $(\mathrm{T})$ versus fluorouracil, epirubicin and cyclophosphamide (FEC) followed by $\mathrm{T}$, all given every 3 weeks or 2 weeks, in node-positive early breast cancer (BC) patients (pts). Final results of the Gruppo Italiano Mammella (GIM)-2 randomized phase III study. SABCS 2013; abstr S5-06.

77 Swain SM, Tang G, Geyer CE, et al.: Definitive results of a phase III adjuvant trial comparing three chemotherapy regimens in women with operable, node-positive breast cancer: the NSABP B-38 trial. J Clin Oncol 2013;31:3197-3204.

78 Budd GT, Barlow WE, Moore HCF, et al.: Comparison of two schedules of paclitaxel for adjuvant therapy of breast cancer. ASCO 2013; abstr.

79 Sparano JA, Wang M, Martino S, et al.: Weekly paclitaxel in the adjuvant treatment of breast cancer. N Engl J Med 2008;358:1663-1671.
80 Moebus V, Jackisch C, Lueck HJ, et al.: Intense dose-dense sequential chemotherapy with epirubicin, paclitaxel, and cyclophosphamide compared with conventionally scheduled chemotherapy in high-risk primary breast cancer: mature results of an AGO phase III study. J Clin Oncol 2010;28: 2874-2880.

81 Romond EH, Perez EA, Bryant J, et al.: Trastuzumab plus adjuvant chemotherapy for operable HER2-positive breast cancer. N Engl J Med 2005; 353:1673-1684.

82 Perez EA, Suman VJ, Davidson NE, et al.: Sequential versus concurrent trastuzumab in adjuvant chemotherapy for breast cancer. J Clin Oncol 2011; 29:4491-4497.

83 Slamon D, Eiermann W, Robert N, et al.; Breast Cancer International Research Group: Adjuvant trastuzumab in HER2-positive breast cancer. N Engl J Med 2011;365:1273-1283.

84 Tolaney SM, Barry WT, Dang CT, et al.: A phase II study of adjuvant paclitaxel (T) and trastuzumab (H) (APT trial) for node-negative, HER2-positive breast cancer (BC). SABCS 2013; abstr S1-04.

85 Donker M, Litière $\mathrm{S}$, Werutsky G, et al.: Breastconserving treatment with or without radiotherapy in ductal carcinoma in situ: 15 -year recurrence rates and outcome after a recurrence, from the EORTC 10853 randomized phase III trial. J Clin Oncol 2013;31:4054-4059.

86 Hughes KS, Schaper LA, Bellon JR, et al.: Lumpectomy plus tamoxifen with or without irradiation in women age 70 years or older with early breast cancer: Long-term follow-up of CALGB 9343. J Clin Oncol 2013;31:2382-2387.

87 Hwang ES, Lichtensztajn DY, Gomez SL, et al.: Survival after lumpectomy and mastectomy for early stage invasive breast cancer: the effect of age and hormone receptor status. Cancer 2013;119: 1402-1411.

88 Souchon R, Sautter-Bihl M-L, Sedlmayer F, et al.: DEGRO practical guidelines: radiotherapy of breast cancer II - radiotherapy of non-invasive neoplasia of the breast. Strahlenther Onkol 2014; 190:8-16.

89 van de Water W, Bastiaannet E, Scholten AN, et al.: Breast-conserving surgery with or without radiotherapy in older breast patients with early stage breast cancer: a systematic review and metaanalysis. Ann Surg Oncol 2014;21:786-794.

90 Aristei C, Palumbo I, Capezzali G, et al.: Outcome of a phase II prospective study on partial breast irradiation with interstitial multi-catheter highdose rate brachytherapy. Radiother Oncol 2013; 108:236-241.

91 Krengli M, Calvo FA, Sedlmayer F, et al.: Clinical and technical characteristics of intraoperative radiotherapy: analysis of the ISIORT-Europe database. Strahlenther Onkol 2013;189:729-737.

92 Leonardi MC, Maisonneuve P, Mastropasqua MG, et al.: Accelerated partial breast irradiation with intraoperative electrons: using GEC-ESTRO recommendations as guidance for patient selection. Radiother Oncol 2013;106:21-27.

93 Olivotto IA, Whelan TJ, Parpia S, et al.: Interim cosmetic and toxicity results from RAPID: a randomised trial of accelerated partial breast irradiation using three-dimensional conformal external beam radiotherapy. J Clin Oncol 2013;31:40384045.

94 Polgar C, Fodor J, Major T, et al.: Breast-conserving therapy with partial or whole breast irradiation: ten-year results of the Budapest randomized trial. Radiother Oncol 2013;108:197-202.
95 Vaidya JS, Wenz F, Bulsara M, et al.: Risk-adapted targeted intraoperative radiotherapy versus whole-breast radiotherapy for breast cancer: 5-year results for local control and overall survival from the TARGIT-A randomised trial. Lancet 2014;383:603-613.

96 Veronesi U, Orecchia R, Maisonneuve P, et al.: Intraoperative radiotherapy versus external radiotherapy for early breast cancer (ELIOT): a randomised controlled equivalence trial. Lancet Oncol 2013;14:1269-1277.

97 Haviland JS, Owen R, Dewar J, et al.: The UK Standardisation of Breast Radiotherapy (START) trials of hypofractionating for treatment of early breast cancer: 10-year follow-up results of two randomized controlled trials. Lancet Oncol 2013;14:1086-1094.

98 Haffty BG, Buchholz TA: Hypofractionated breast radiation: preferred standard of care? Lancet Oncol 2013;14:1032-1034.

99 Herbert C, Nichol A, Olivotto I, et al.: The impact of hypofractionated whole breast radiotherapy on local relapse in patients with grade 3 early breast cancer: a population-based cohort study. Int J Radiat Oncol 2012;82:2086-2092.

100 Whelan TJ, Pignol JP, Levine MN, et al.: Longterm results of hypofractionated radiation therapy for breast cancer. N Engl J Med 2010;362:513-520.

101 Sedlmayer F, Sautter-Bihl ML, Budach W, et al DEGRO practical guidelines: radiotherapy of breast cancer I: Radiotherapy following breast conserving therapy for invasive breast cancer. Strahlenther Onkol 2013;189:825-833.

102 Chen X, Yu X, Chen J, et al.: Radiotherapy can improve the disease-free survival rate in triplenegative breast cancer patients with $\mathrm{T} 1-\mathrm{T} 2$ disease and one to three positive lymph nodes after mastectomy. Oncologist 2013;18:141-147.

103 Li Y, Moran MS, Huo Q, et al.: Post-mastectomy radiotherapy for breast cancer patients with $\mathrm{T} 1-$ T2 and 1-3 positive lymph nodes: a meta-analysis. PLoS One 2013;8:e81765.

104 Moo TA, McMillan R, Lee M, et al.: Selection criteria for postmastectomy radiotherapy in T1T2 tumors with 1 to 3 positive lymph nodes. Ann Surg Oncol 2013;20:3169-3174.

105 Shim SJ, Park W, Huh SJ, et al.: The role of postmastectomy radiation therapy after neoadjuvant chemotherapy in clinical stage II-III breast cancer patients with pN0: a multicenter, retrospective study (KROG 12-05). Int J Radiat Oncol Biol Phys 2014;88:65-72.

106 Liu D, Chen Y, Deng M, et al.: Lymph node ratio and breast cancer prognosis: a meta-analysis. Breast Cancer 2014;21:1-9.

107 Saquib J, Parker BA, Natarajan L, et al.: Prognosis following the use of complementary and alternative medicine in women diagnosed with breast cancer. Complement Ther Med 2012;20:283-290.

108 Qi CJ, Ning YL, Han YS, et al.: Autologous dendritic cell vaccine for estrogen receptor (ER)/progestin receptor (PR) double-negative breast cancer. Cancer Immunol Immunother 2012;61:1415-1424.

109 Montero AJ, Diaz-Montero CM, Deutsch YE, et al.: Phase 2 study of neoadjuvant treatment with NOV-002 in combination with doxorubicin and cyclophosphamide followed by docetaxel in patients with HER-2 negative clinical stage II-IIIc breast cancer. Breast Cancer Res Treat 2012; 132:215-223.

110 Adams S, Kozhaya L, Martiniuk F, et al.: Topical TLR7 agonist imiquimod can induce immune-mediated rejection of skin metastases in patients with breast cancer. Clin Cancer Res 2012;18:6748-6757. 